\title{
ANÁLISIS DE CASOS \\ Inclusión de estudiantes con necesidades educativas especiales en contexto rural
}

\section{Inclusion of students with special educational needs in a rural context}

\section{Inclusão de alunos com necessidades educacionais especiais em contexto rural}

\author{
Giovanna Hernández Cortés \\ Trabajadora Social, Magister en Trabajo Social \\ Graduada del Magister en Trabajo Social PUCCH \\ Santiago, Chile \\ ghernandez2@uc.cl \\ https://orcid.org/0000-0003-1622-4300
}

\author{
Alejandra Rasse Figueroa \\ Socióloga, Magister en Sociología, Doctora en Arquitectura y Estudios Urbanos \\ Escuela de Trabajo Social PUCCH, Centro de Desarrollo Urbano Sustentable CEDEUS \\ Santiago, Chile \\ Autor para correspondencia: arasse@uc.cl \\ https://orcid.org/0000-0003-0625-8021
}

\begin{abstract}
Resumen
Durante los últimos años, la inclusión escolar ha sido objeto de múltiples estudios y políticas públicas. No obstante, es habitual ver que se trabaja sobre el supuesto de escuelas con caracteristicas urbanas, con estudiantes diferenciados por niveles, y en las que existe, en alguna medida, una comunidad pedagógica que posibilita el apoyo en el aula y la reflexión docente. Esto deja fuera del foco de la discusión sobre inclusión a la escuela rural multigrado. A través de un estudio de caso cualitativo, este artículo analiza la forma en que las escuelas rurales multigrado de una comuna del norte chico de Chile abordan la inclusión de estudiantes con necesidades educativas especiales. Los resultados muestran que la aplicación de políticas con enfoque urbano en sectores rurales, generan diversas problemáticas que profundizan las desigualdades territoriales, y revelan hitos críticos necesarios de ser abordados: la integralidad de la inclusión, la continuidad de los procesos inclusivos, y los momentos de egreso y transición escolar.
\end{abstract}

Palabras clave: Inclusión escolar, ruralidad, necesidades educativas especiales, Escuela Rural.

\footnotetext{
Abstract

In recent years, multiple studies and public policies had addressed school inclusion. However, it is common to see that we work on the assumption of schools with urban characteristics, with students differentiated by levels, and in which there is, to some extent, a pedagogical community that allows both classroom support and teacher reflection. This leaves the multigrade rural school out of the inclusion debate. Through a qualitative case study, this paper shows how the inclusion of students with special educational needs is addressed in multigrade rural schools in a commune in the northern part of Chile. The results show that the application of policies with an urban focus in rural sectors (i) deepen territorial inequalities, (ii) create new problems, and (iii) reveal critical issues that need to be addressed: graduation processes, transition to high school, inclusion
} 
multidimensionality, and the continuity of inclusive processes as a whole.

Key words: School Inclusion, rurality, special educational needs, Rural School.

\begin{abstract}
Resumo
Nos últimos anos, a inclusão escolar tem sido objeto de múltiplos estudos e políticas públicas. No obstante, es habitual ver que se trabaja sobre el supuesto de escuelas con caracteristicas urbanas, con estudiantes diferenciados por niveles, y en las que existe, en alguna medida, una comunidad pedagógica que posibilita el apoyo en el aula y la reflexión docente. Isso deixa a escola rural multisseriada fora do foco da discussão sobre inclusão. Por meio de um estudo de caso qualitativo, este artigo apresenta como se aborda a inclusão de alunos com necessidades educacionais especiais em escolas multisseriadas rurais de uma comuna do norte do Chile. Os resultados mostram que a aplicação de políticas com enfoque urbano nos setores rurais geram diversos problemas e aprofundam as desigualdades territoriais, e revelam marcos críticos que precisam ser trabalhados: a integralidade da inclusão, a continuidade dos processos inclusivos e os momentos de graduação. e transição escolar.
\end{abstract}

Palavras-chave: Inclusão escolar, ruralidade, necessidades educacionais especiais, Escola Rural.

\section{Las necesidades educativas especiales en el contexto de la escuela rural}

Las políticas educativas se han reformado constantemente a través del tiempo, en un proceso que se relaciona con los cambios de nuestro entorno social, cultural, político, económico e institucional. (Aziz, 2018). No obstante, la educación rural ha permanecido marginada de las discusiones de política educativa actuales, a pesar de sus evidentes carencias a nivel de recursos humanos y materiales, y de la brecha de resultados de aprendizaje y completitud de estudios al compararla con la situación urbana (MINEDUC, 2017). Esta situación se comprende en un marco mayor, en tanto las políticas estatales en Chile han sido diseñadas sobre la consideración de un país urbano, en desmedro de quienes habitan en zonas rurales (Muñozo, Núñez, Niculcar, González, Costa, Ascorra y Shaun, 2020).

Esta situación se replica cuando se observan las políticas especificas implementadas para promover la inclusión escolar. Si bien el Ministerio de Educación ha buscado generar iniciativas que permitan avanzar hacia un sistema que reconozca y garantice de mejor manera las oportunidades para los estudiantes con necesidades educativas especiales, independiente de su contexto de origen y del establecimiento al que asistan (MINEDUC, 2021a), existe evidencia que da cuenta de la persistencia de las inequidades territoriales. Un ejemplo de ello es la cobertura del programa de integración escolar (en adelante PIE), orientado a la inclusión de niños y niñas que presentan Necesi- dades Educativas Especiales. En el año 2019, un 65\% de las escuelas urbanas cuentan con Programa de Integración Escolar (PIE), en contraste con el 35\% de escuelas ubicadas en sectores rurales que cuenta con este programa (MINEDUC, 2021b).

Las escuelas rurales son las que presentan mayores dificultades para la implementación de la política PIE, dado el diseño centralista del programa, que no considera adecuaciones eficaces para los recintos educacionales que se encuentran en lugares alejados. Esto se traduce en inequidades territoriales que coartan el desarrollo de los niños y niñas, partiendo de la base de las bajas posibilidades de que reciban un diagnóstico que le permita a la escuela solicitar apoyo al PIE, debido a una escasez de profesionales especializados y a la falta de recursos para contratarlos cuando se trata de una escuela pequeña (Agencia de Calidad de la Educación, 2016; López, 2014).

En este contexto, la educación rural multigrado enfrenta, como todas las escuelas, el desafío de garantizar aprendizajes de calidad e igualdad de oportunidades a aquellos estudiantes que presentan necesidades educativas especiales (MINEDUC, 2021c), pero en un escenario social, territorial e institucional totalmente distinto al de una escuela urbana. En la práctica, los estudiantes rurales son vistos desde la carencia por su condición socioterritorial y distanciamiento respecto del "ideal urbano", ampliando así aún más la brecha desigual y la falta de reconocimiento como sujetos de derecho (López, 2014; Díaz, Osses y Muñoz, 2016; PNUD, 2008). 


\section{El programa PIE}

El programa de integración escolar (en adelante PIE), es una estrategia inclusiva del sistema educacional que busca contribuir y favorecer los aprendizajes en la sala de clases y la participación de todos y cada uno de los estudiantes, especialmente de aquellos que presentan Necesidades Educativas Especiales (NEE), a través de estrategias pedagógicas diversificadas, recursos humanos especializados, acompañamiento para los docentes y material educativos adecuados (Marfán, Castillo, González y Ferreira, 2013).

Los PIE se pueden desarrollar en establecimientos educacionales o en un grupo de ellos, como sucede en los PIE comunales, en ambos casos, los sostenedores y equipos directivos tienen la función de liderar las distintas fases de planificación, implementación y evaluación. El equipo profesional PIE está compuesto multidisciplinariamente por Profesores Especialistas y por Profesionales Asistentes de la Educación como lo son los Psicopedagogos, Psicólogos, Fonoaudiólogos, Trabajadores Sociales, entre otros, existiendo un trabajo colaborativo entre los docentes y los asistentes descritos. "De este modo el PIE se constituye en un conjunto de recursos y apoyos para los centros educativos, que en el aula se traducen en estrategias pedagógicas diversificadas, recursos humanos especializados, capacitación para los docentes y materiales educativos pertinentes a las necesidades de los estudiantes" (MINEDUC, 2016, p.9).

En la práctica, el PIE funciona como un subsidio extra que se asigna a la escuela por cada estudiante diagnosticado con alguna de las problemáticas consideradas por el PIE como NEE, para ser destinado a la constitución de un equipo profesional que de apoyo a este grupo de estudiantes en sus procesos de aprendizaje, lo que se realiza en algunos casos al interior del aula durante las clases regulares, y en otros, fuera del aula, en talleres específicos. Esto da al programa un fuerte énfasis psico-médico, creando una especie de "etiquetaje" que genera discriminación y desigualdad dentro del aula, es decir, "prescribe un modelo de integración consistente en una atención individual de la discapacidad y a su vez descansa sobre una visión patológica de la diferencia” (López et al., 2014, p. 14).

Las condiciones de la escuela rural multigrado, en términos de accesibilidad, número de estudiantes, características de los estudiantes y sus familias, acceso a la salud (y en especial, a médicos especialistas), y numero de recintos disponibles en la escuela para el trabajo con estudiantes, generan una serie de dificultades en la aplicación práctica de un programa diseñado bajo el modelo recién descrito. Pese a las evidentes diferencias con el contexto urbano, existe poca investigación en torno a la aplicación de las políticas educativas en el medio rural. Contar con información adicional que permita seguir mejorando los resultados educativos y la viabilidad de la implementación de las políticas educativas en contexto rural, continúa siendo una necesidad (OCDE, 2016).

\section{Análisis de caso: funcionamiento del PIE en una comuna rural del norte chico en Chile}

En este marco, este trabajo analiza la forma en que se aborda en escuelas rurales multigrado la inclusión de estudiantes con necesidades educativas especiales. Para esto, se desarrollo un estudio cualitativo de caso, con foco en una comuna rural del norte chico, que cuenta con tres escuelas rurales multigrados. Se entrevistó a la totalidad de los profesionales de trato directo con estudiantes, más sus jefaturas directas. En total fueron 7 personas que participaron en entrevistas semiestructuradas en 3 grupos: Docentes, Profesionales PIE y Jefaturas. Todas las entrevistas fueron transcritas de forma literal, y analizadas con categorías temáticas emergentes.

La comuna en estudio se ubica hacia la cordillera del norte chico, y su población comunal es de poco más de 5.000 habitantes. La economía local de la comuna, se desarrolla mayoritariamente en torno a la agricultura; también predomina con la crianza de ovejas y cabras, siendo una carga laboral en la que participan las familias, incluyendo a niños y niñas. Presenta uno de los índices de Desarrollo Humano (IDH) más bajos de toda la zona. Asimismo, se muestra como la comuna con más baja escolaridad, mayor daño ambiental, contaminación y aislamiento, menor alfabetización y la que representa una mayor tasa de mortalidad infantil (INE, 2021). El acceso a bienes y servicios es limitado, no existen bancos, supermercados o tiendas comerciales, y los más cercanos se encuentran en la zona urbana más próxima, que queda a 3 horas de distancia en bus desde las localidades de las escuelas 2 y 3 , y, a 2 horas en bus desde la localidad de la escuela 1.

El equipo PIE de la comuna tiene incorporadas a sus nóminas 12 estudiantes con NEE de un total de 28 
matrículas que suman las 3 escuelas. Los estudiantes que presentan NEE, según los entrevistados, por lo general provienen de familias vulnerables en múltiples sentidos, existiendo casos en los que sus propios padres son analfabetos y/o mantienen un diagnóstico de trastorno cognitivo severo. Los profesionales PIE, al ser itinerantes, solo viajan a cada escuela a realizar sus terapias 1 o 2 veces por semana.

De acuerdo a las entrevistas realizadas, es posible identificar 6 dimensiones críticas en términos de la aplicación del PIE en el contexto recién descrito:

\section{Situación territorial}

El contexto territorial en el que se encuentran insertas las escuelas rurales estudiadas se muestra como un factor que complejiza el abordaje de la inclusión en estudiantes con NEE. La gran dispersión geográfica genera aislamiento entre las comunidades y una falta de instancias de socialización para los estudiantes y sus familias que vayan más allá de la escuela.

En este marco, la diferencia respecto de la situación urbana resulta tanto una desventaja, como un recurso. En el fondo, el aislamiento resulta una desventaja a la hora de acceder a redes (comunitarias, de salud, educativas, etc.) que colaboren en la inclusión de estos estudiantes, así como en la imposibilidad de la presencia constante del apoyo del PIE en la escuela (como si ocurre en escuelas de mayor matricula en zonas urbanas. Pero el aislamiento se vuelve un factor protector, en tanto estos niños y niñas se desenvuelven en un entorno en que son conocidos por todos y apoyados no solo por sus familias sino también por amistades y vecinos.

\section{El componente afectivo}

Las acciones que se llevan a cabo en las escuelas rurales, varían entre profesionales de acuerdo a su comprensión de la inclusión; desde modelos individualistas (tradicionales y médicos), hasta las miradas más innovadoras que predominan en la actualidad, como lo es con un abordaje mediante la afectividad, aceptación y valoración de diferencias (Reverón, Rodríguez y Hernández, 2017). Los entrevistados que sostienen esta última postura incorporan elementos como la empatía y establecimiento de fuertes vínculos afectivos que persistan a través del tiempo.
De acuerdo a los entrevistados, esta visión afectiva puede establecerse con mayor facilidad en el ámbito rural, a razón de las posibilidades de cercanía que se producen en los contextos rurales y al bajo número de estudiantes que asisten a las escuelas rurales unidocentes, donde se logra establecer con mayor facilidad vínculos afectivos con los estudiantes y sus familias, conocer sus historias de vida y la realidad sobre la cual se desarrollan. Es habitual que los profesores conozcan no solo al estudiante y apoderado, sino a otros miembros de la familia, el lugar donde viven, e incluso a miembros de la comunidad que sirven de apoyo al proceso educativo. Ello se muestra como una diferencia respecto del medio urbano, donde los profesores declaran dificultades para el manejo afectivo y emocional de los estudiantes que son atendidos por el PIE (Tamayo et al, 2018).

\section{La reprobación como mecanismo alternativo de reparación}

Dado que estas escuelas cubren solo la enseñanza básica (siendo necesario viajar hacia zonas urbanas para cursar la enseñanza media), algunos docentes identifican la reprobación como un medio para poder continuar trabajando y progresando con los estudiantes con NNE, logrando en cierta medida cumplir, en tiempos más largos, con los objetivos académicos propuestos de acuerdo al diagnóstico que los estudiantes presenten. Este tipo de estrategias es contraria a las indicaciones ministeriales respecto a la adecuación curricular para NNA con NEE.

Aquí el factor territorial vuelve a emerger, toda vez que hay que tener en cuenta que el egreso de un estudiante con NEE de su escuela significa un cambio rotundo en su estilo de vida, sobre todo considerando las amplias distancias que existen desde un lugar a otro. Ya no se trata de la escuela de su localidad donde interactúa con sus compañeros que conoce desde primero básico; ahora el estudiante debe aventurarse a asistir a una escuela que se encuentra en el centro de la comuna, es decir, cerca de 2 horas de trayecto en un bus escolar de ida y 2 horas de vuelta. Ya no existe la sala multigrado donde un único profesor dicta las clases, ahora es un colegio dotado de infraestructura diferente y esquemas completamente distintos a los que solía conocer. Asimismo, pierde los lazos de confianza que quizás pudo haber establecido con el profesor o el profesional PIE, que se disuelven ante este abrupto cambio. 
Adicionalmente, al estar el programa pensado desde una lógica individual y más cercana a lo médico, no existe una entidad que se encargue de facilitar la transición de estos estudiantes hacia un nuevo establecimiento educacional, por ejemplo, seguimiento psicosocial, coordinaciones con el nuevo equipo PIE que lo atenderá, o simplemente un proceso de traspaso entre los mismos profesores.

En este marco, los apoderados optan porque sus hijos continúen repitiendo de curso para que no egresen de la escuela, y no tener que enfrentarlos a esta realidad que pareciera ser tan compleja. En la práctica, el resultado es que se adapta al estudiante a las condiciones institucionales "posibles", manteniéndolo como repitente en la escuela, porque no hay alternativa de un proceso adecuado para él, perdiéndose el sentido de inclusión de la diversidad y la noción de capacidades diferentes.

\section{Inadecuación a localidades lejanas o aisladas}

El programa PIE pierde pertinencia producto de las distancias entre localidades en contextos rurales. Al estar pensado como apoyo a determinados estudiantes que presentan NEE, en territorios rurales la concentración de estos niños en una determinada escuela no permite su presencia permanente, lo que obliga a la itinerancia en rondas del equipo PIE. Esto dificulta el trabajo, en la medida en que los profesionales están viajando entre 4 y 6 horas al día. Asimismo, la itinerancia lleva a que los equipos PIE no cuenten con un lugar de trabajo propio, ni con recursos educativos en cada escuela que visitan, lo que dificulta el trabajo con los estudiantes. Todas estas condiciones dificultan la retención de los profesionales, generando rotación y discontinuidad en los procesos.

Por otra parte, los docentes de las escuelas rurales no cuentan con formación específica para apoyar a los estudiantes con NEE de forma autónoma, lo que unido a la itinerancia de los profesionales PIE y a la escases de recursos para el aprendizaje en las escuelas, dificulta la instalación de procesos de enseñanza aprendizaje adaptados a los estudiantes que requieren de adecuaciones.

\section{Inclusión Integral}

Los diversos actores que participaron de este estudio relevan la importancia de desarrollar una visión global sobre el proceso inclusivo de NNA: este trabajo no termina con el egreso de la escuela rural unidocente, y por ende, las prácticas y políticas inclusivas no se pueden reducir o delimitar a un establecimiento educacional en particular. Al contrario, estos estudiantes requieren un acompañamiento integral a lo largo de su vida, incluso más allá de lo escolar. Como los mismos sujetos de investigación lo refieren, los estudiantes con NEE que no logran ser incluidos, el día de mañana serán adultos que experimentarán procesos de exclusión social y ocupacional. Esto se agudiza en el contexto rural, dadas las desigualdades territoriales y brechas de bienestar que los afectan.

En este sentido, el trabajo de inclusión no puede recaer sólo en el PIE, sino que para resguardar los derechos de estos NNA, debe existir una política pública integral que genere las redes y apoyos necesarios. Si bien dichas redes son mas difíciles de establecer en el contexto rural, son esenciales, especialmente en el egreso del estudiante con NEE de su establecimiento.

\section{Medicalización y "estudiantes solidarios"}

Dado que la aplicación del PIE depende del diagnóstico de los estudiantes como afectos a alguna NEE, su modelo es individual, es decir, no se aplica a la comunidad educativa como un todo, sino solo a aquellos estudiantes que poseen un diagnostico de NEE. Sin embargo, las dificultades de accesibilidad a especialistas en el medio rural lleva a que sea común que haya NNA que, presentando todos los indicios de requerir apoyo especializado, no cuentan con un diagnóstico que permita incorporarlos en el programa. Los entrevistados relatan que, por motivos éticos, estos estudiantes de todos modos son apoyados, bajo el concepto de "estudiantes solidarios". Esto representa una sobrecarga de trabajo para los profesionales del PIE, pero también una reducción en el tiempo efectivo de trabajo con los demás NNA.

En la práctica, este enfoque individual y medico subestima la magnitud de los apoyos requeridos en las escuelas rurales, y en consecuencia, profundiza las desventajas y contribuye a reproducir las condiciones de precariedad y pobreza que la comuna tiene.

\section{Conclusiones y recomendaciones}

En conclusión, el PIE es un programa que ha sido diseñado desde una perspectiva urbana y poniendo énfasis en el componente médico y la atención individual. Estos elementos comprometen fuertemente su 
pertinencia en entornos rurales, producto de la baja concentración de alumnos, las largas distancias, las dificultades para articular con redes de salud y otros apoyos públicos, las desventajas territoriales, y las características socioculturales de las familias. Todo lo anterior genera una subestimación de las necesidades de apoyo de las escuelas rurales multigrado, y el despliegue de apoyos intermitentes en contextos sociales, familiares e institucionales en que se vuelve difícil asegurar la continuidad de los procesos educativos de los NNA con NEE. Esto compromete la posibilidad de dar garantía de derechos a los NNA en entornos rurales, y contribuye a la profundización de las desigualdades educativas y territoriales.

Desde nuestra perspectiva, y según lo expuesto, para avanzar hacia el aseguramiento del derecho de educación en estudiantes con NEE en contexto rural, resulta necesario:

1.- Promover el sentido de responsabilidad de co-garante de derechos de la infancia a la comunidad educativa completa, y si es posible, ampliar esto a la comunidad territorial.

2.- Mejorar la especialización de los docentes de escuelas rurales multigrado en materia de inclusión y NEE, considerando que los apoyos del equipo del PIE comunal son itinerantes y que las características socioculturales y ocupacionales de las familias les dificultan ejercer un apoyo activo en el proceso educativo. Esto permitiría mejorar la pertinencia y oportunidad de las adecuaciones curriculares y de evaluación, con altas implicancias en el proceso formativo de los NNA.

4.- Es menester que los equipos PIE comunales cuenten con un profesional de Trabajo Social en el grupo, que gestione los procesos de inclusión en redes, relación familia - escuela - comunidad, y desarrolle los vínculos interescuela necesarios para dar fluidez a las transiciones. Se considera que el incorporar a un/a trabajador/a social como miembro obligatorio del estamento profesional de los asistentes de la educación rural, se logrará facilitar el proceso de inclusión integral.

5.- Se muestra necesario el poder inyectar mayores recursos a las escuelas rurales. Si bien nuestro sistema educativo se basa en un financiamiento que se calcula en base a un voucher individual por NNA, en la práctica esto implica situar en una posición de permanente desventaja a las escuelas rurales.

6. Pensar el PIE como un programa con foco en la comunidad educativa como un todo, y no sólo en estudiantes individuales, de modo de poner fin al concepto "estudiantes solidarios".

7.- Evaluar alternativas de itinerancia que garanticen y asignen un mayor tiempo de trabajo con los estudiantes, los docentes y familias, toda vez que la falta de acompañamiento durante la semana del equipo PIE compromete las posibilidades de mejora.

Más allá de estas propuestas, resulta imprescindible hacer visible que el diseño de políticas nacionales desde una perspectiva urbana profundiza las desigualdades territoriales, y el abordaje individual de problemáticas que son esencialmente comunitarias promueve, en el corto plazo, el etiquetaje, y en el largo plazo, el desencadenamiento de procesos de exclusión social que comprometen las posibilidades de inclusión futura de los NNA que se busca apoyar.

\section{Referencias}

AGENCIA DE CALIDAD DE LA EDUCACIÓN (2016). CARACTERÍSTICAS DEL LIDERAZGO DIRECTIVO EN ESCUELAS RURALES EFECTIVAS. Editorial Agencia de Calidad de la Educación. http:// archivos.agenciaeducacion.cl/liderazgo-motivacionlectora/Estudio_Liderazgo_Rural.pdf

AZIZ, C. (2018). Evolución e implementación de las políticas educativas en Chile. Nota técnica No 2 LIDERESEDUCATIVOS, Editorial Centro de Liderazgo para la Mejora Escolar. https://www.lidereseducativos.cl/wp-content/ uploads/2018/06/NT2_L6_C.A_Evolucio\%CC\%81ne-implementacio\%CC\%81n-de-las-poli\%CC\%81ticaseducativas-en-Chile.pdf

DÍAZ FUENTES, R., OSSES BUSTINGORRY, S., \& MUÑOZ NAVARRO, S. (2016). Factores e interacciones del proceso de enseñanza-aprendizaje en contextos rurales de la Araucanía, Chile. Estudios pedagógicos 
(Valdivia), 42(3), 111-128. https://scielo.conicyt.cl/ pdf/estped/v42n3/art06.pdf

INSTITUTO NACIONAL DE ESTADISTICAS, INE (31 DE JULIO DE 2021). CENSO DE POBLACIÓN Y VIVIENDA 2017. https://www.ine.cl/estadisticas/ sociales/censos-de-poblacion-y-vivienda

LÓPEZ, V. (2014). Barreras culturales para la Inclusión: políticas y prácticas de Integración en Chile. Revista de Educación, 363, 256-281. http://www.revistaeducacion.educacion.es/doi/363_180.pdf

MARFÁN, J., CASTILlO, P., GONZÁLEZ, R., \& FERREIRA, I. (2013). Análisis de la implementación de los programas de integración escolar (PIE) en establecimientos que han incorporado estudiantes con necesidades educativas especiales transitorias (NEET). Editorial Fundación Chile. https://especial.mineduc.cl/ wp-content/uploads/sites/31/2016/08/Resumen_Estudio_ImplementacionPIE_2013.pdf

MINISTERIO DE EDUCACIÓN, MINEDUC (2017). REVISIÓN DE LAS POLÍTICAS EDUCATIVAS EN CHILE DESDE 2004 A 2016: INFORME NACIONAL. Editorial Centro de Estudios MINEDUC.

HTTPS://BIBLIOTECADIGITAL.MINEDUC.cl/handle/20.500.12365/4468

MINISTERIO DE EDUCACIÓN, MINEDUC (31 DE JULIO DE 2021A). DIRECTORIO ESTABLECIMIENTOS CON PROGRAMA DE INTEGRACIÓN ESCOLAR (PIE). https://especial.mineduc.cl/directorio-de-establecimientos/ MINISTERIO DE EDUCACIÓN, MINEDUC (31 DE JULIO DE 2021B). CENTRO DE DATOS ABIERTOS. http://datosabiertos.mineduc.cl/
MINISTERIO DE EDUCACIÓN, MINEDUC (31 DE JULIO DE 2021C). OBJETIVOS Y ÉNFASIS DE TRABAJO. Educación Rural. https://rural.mineduc.cl/objetivos-y-enfasis-de-trabajo-2018/

MUÑOZO, C.N., NICULCAR, B.G., COSTA, P.A., \& GRECH, S. (2020). Contar Para Comprender: Cierre De Escuelas Rurales Municipales en Chile y sus implicancias para las Comunidades. Educ. Soc., Campinas, v. 41, s/p. https://doi.org/10.1590/es.215922

ORGANIZACIÓN PARA LA COOPERACIÓN Y EL DESARROLLO ECONÓMICO, OCDE (2016). ESTUDIOS DE POLÍTICA RURAL. Editorial OCDE. https://www.odepa.gob.cl/wp-content/ uploads/2018/10/Estudios-de-Poli\%CC\%81tica-RuralChile-OCDE.pdf

PROGRAMA DE LAS NACIONES UNIDAS PARA EL DESARROLLO, PNUD (2008). INFORME SOBRE DESARROLLO HUMANO EN CHILE RURAL: SEIS MILLONES POR NUEVOS CAMINOS. Santiago: PNUD.

REVERÓN, C. A., RODRIGUEZ, V. L., Y HERNÁNDEZ, C. H. (2017). La inclusión escolar de los niños con necesidades especiales. Canarias: Formación y estudios.

TAMAYO, M. ET AL. (2018): Programa de Integración Escolar en Chile: brechas y desafíos para la implementación de un programa de educación inclusiva. Revista Española de Discapacidad, 6 (I): 161-179. https://doi. org/10.5569/2340-5104.06.01.08 


\section{TABLA 1. MODALIDADES DE LOS BENEFICIOS PENITENCIARIOS}

\begin{tabular}{|c|c|}
\hline \multicolumn{2}{|r|}{ Ley Nacional de Ejecución Penal (2016) } \\
\hline \multirow{8}{*}{$\begin{array}{l}\text { Artículo } 136 \text { y } 137 . \\
\text { Libertad condicionada }\end{array}$} & Los requisitos son: \\
\hline & I. Que no se le haya dictado diversa sentencia condenatoria firme; \\
\hline & $\begin{array}{l}\text { II. Que no exista un riesgo objetivo y razonable en su externamiento para la víctima u ofendido, los testigos } \\
\text { que depusieron en su contra y para la sociedad; }\end{array}$ \\
\hline & III. Haber tenido buena conducta durante su internamiento; \\
\hline & IV. Haber cumplido satisfactoriamente con el Plan de Actividades al día de la solicitud; \\
\hline & $\begin{array}{l}\text { V. Haber cubierto la reparación del daño y la multa, en las modalidades y con las excepciones establecidas } \\
\text { en esta Ley; }\end{array}$ \\
\hline & VI. No estar sujeto a otro proceso penal del fuero común o federal por delito que amerite prisión preventiva, y \\
\hline & VII. Que se haya cumplido con la mitad de la pena tratándose de delitos dolosos. \\
\hline \multirow[t]{8}{*}{$\begin{array}{l}\text { Artículo 14l. Libertad } \\
\text { anticipada }\end{array}$} & $\begin{array}{l}\text { El otorgamiento de la libertad anticipada extingue la pena de prisión y otorga libertad al sentenciado. Los } \\
\text { siguientes requisitos son: }\end{array}$ \\
\hline & I. Que no se le haya dictado diversa sentencia condenatoria firme; \\
\hline & $\begin{array}{l}\text { II. Que no exista un riesgo objetivo y razonable en su externamiento para la víctima u ofendido, los testigos } \\
\text { que depusieron en su contra y para la sociedad; }\end{array}$ \\
\hline & III. Haber tenido buena conducta durante su internamiento; \\
\hline & IV. Haber cumplido con el Plan de Actividades al día de la solicitud; \\
\hline & V. Haber cubierto la reparación del daño y la multa, en su caso; \\
\hline & $\begin{array}{l}\text { VI. No estar sujeto a otro proceso penal del fuero común o federal por delito que amerite prisión preventiva } \\
\text { oficiosa, y }\end{array}$ \\
\hline & $\begin{array}{l}\text { VII. Que hayan cumplido el setenta por ciento de la pena impuesta en los delitos dolosos o la mitad de la } \\
\text { pena tratándose de delitos culposos. }\end{array}$ \\
\hline \multirow[t]{5}{*}{$\begin{array}{l}\text { Artículo } 144 \text {. Sustitución } \\
\text { de la pena }\end{array}$} & $\begin{array}{l}\text { El Juez de Ejecución podrá sustituir la pena privativa de la libertad por alguna pena o medida de seguridad } \\
\text { no privativa de la libertad, previstas en esta Ley cuando durante el periodo de ejecución se actualicen los } \\
\text { siguientes supuestos: }\end{array}$ \\
\hline & $\begin{array}{l}\text { I. Cuando se busque la protección de las hijas e hijos de personas privadas de la libertad, siempre que éstos } \\
\text { sean menores de } 12 \text { años de edad o tengan una condición de discapacidad que no les permita valerse por } \\
\text { sí mismos. Esto cuando la persona privada de la libertad sea su cuidadora principal o única cuidadora, de } \\
\text { acuerdo con lo dispuesto en esta Ley. }\end{array}$ \\
\hline & $\begin{array}{l}\text { II. Cuando la permanencia de la persona sentenciada con la hija, hijo o persona con discapacidad no re- } \\
\text { presenta un riesgo objetivo para aquellos. }\end{array}$ \\
\hline & $\begin{array}{l}\text { III. Cuando esta fuere innecesaria o incompatible con las condiciones de la persona privada de la libertad } \\
\text { por senilidad, edad avanzada, o su grave estado de salud, en los casos regulados en la legislación penal } \\
\text { sustantiva, de acuerdo con las reglas de competencia establecidas en esta Ley. }\end{array}$ \\
\hline & $\begin{array}{l}\text { IV. Cuando, en términos de la implementación de programas de tratamiento de adicciones, reinserción en } \\
\text { libertad, justicia colaborativa o restitutiva, política criminal o trabajo comunitario, el Juez de Ejecución } \\
\text { reciba de la Autoridad Penitenciaria o de la autoridad de supervisión un informe sobre la conveniencia } \\
\text { para aplicar la medida y si el sentenciado no representa un riesgo objetivo y razonable para la víctima u } \\
\text { ofendido, los testigos que depusieron en su contra y para la sociedad. Dicha autoridad deberá fungir como } \\
\text { aval para la sustitución. }\end{array}$ \\
\hline $\begin{array}{l}\text { Artículo } 145 \text {. Permisos } \\
\text { extraordinarios de salida } \\
\text { por razones humanitarias }\end{array}$ & $\begin{array}{l}\text { La persona privada de su libertad podrá solicitar al Juez de Ejecución un permiso extraordinario de salida } \\
\text { cuando se justifique por enfermedad terminal, fallecimiento de un pariente consanguíneo en línea ascen- } \\
\text { diente o descendiente de primer grado, cónyuge, concubina o concubinario, o socio conviviente. Esta } \\
\text { medida no aplicará para las personas privadas de su libertad por delincuencia organizada o aquellas sujetas } \\
\text { a medidas especiales de seguridad. El permiso será otorgado siempre y cuando implique un traslado en } \\
\text { la misma localidad, o dentro de un radio razonable, condicionado a que este sea viable y materialmente } \\
\text { posible. }\end{array}$ \\
\hline
\end{tabular}


Artículo 146.

Preliberación por criterios de política penitenciaria
De acuerdo a alguno de los siguientes criterios:

I. Se trate de un delito cuya pena máxima sea de cinco años de prisión, siempre que el delito no se haya cometido con violencia;

II. Se trate de delitos de contenido patrimonial cometidos sin violencia sobre las personas o de delitos culposos;

III. Por motivos humanitarios cuando se trate de personas sentenciadas adultas mayores, portadoras de una enfermedad crónico-degenerativa o terminal, independientemente del tiempo que lleven compurgando o les falte por compurgar de la sentencia;

IV. Cuando se trate de personas sentenciadas que hayan colaborado con la procuración de justicia o la Autoridad Penitenciaria, y no hayan sido acreedoras a otra medida de liberación;

V. Cuando se trate de delitos de cuyo bien jurídico sea titular la federación o la entidad federativa, o aquellos en que corresponda extender el perdón a estos;

VI. Cuando la continuidad de la aplicación de la pena sea irrelevante para los fines de la reinserción del sentenciado a la sociedad o prevenir la reincidencia.

Fuente: Elaboración propia a partir de la Ley Nacional de Ejecución Penal (2021). 